\title{
Epidemiology of gastroenteropancreatic neuroendocrine tumours
}

\author{
M. Fraenkel, MD, Lecturer ${ }^{\mathrm{a}, *}$, M.K. Kim, MD, MSc, \\ Director, Assistant Professor ${ }^{\mathrm{b}, 1}$, A. Faggiano, MD, PhD, \\ Research Fellow $^{\text {c,d,2 }}$, G.D. Valk, MD, PhD, Senior Researcher ${ }^{\mathrm{e}, 3}$

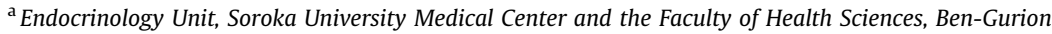 \\ University of the Negev, Yitzchag Rager, Beer Sheva, Israel \\ ${ }^{\mathrm{b}}$ Division of Gastroenterology, Department of Medicine, Mount Sinai School of Medicine, New York, NY, USA \\ ${ }^{\mathrm{c}}$ Dept of Clinical Medicine and Surgery, Federico II University of Naples, Napoli, Italy \\ ${ }^{\mathrm{d}}$ Endocrinology, National Cancer Institute, Fondazione G. Pascale, Naples, Italy \\ ${ }^{\mathrm{e}}$ Universtity Medical Center Utrecht, Dept. of Internal Medicine, PO Box 85500, 3508 GA Utrecht, The \\ Netherlands
}

Keywords:

Gastroenteropancreatic neuroendocrine tumours

Carcinoid

Epidemiology

Incidence

Survival

\begin{abstract}
A B S T R A C T
Gastroenteropancreatic neuroendocrine tumours are a heterogeneous group of tumours arising from diffuse endocrine cells, causing unique clinical syndromes. These tumours, formerly named carcinoid, can involve any part of the gastrointestinal tract and the endocrine pancreas and have a wide range of malignant potential: from benign to poorly differentiated tumours. In this review we will summarize the data available on the epidemiology of gastroenteropancreatic tumours as it is reported from around the world. This includes annual incidence rates at the various anatomic sites, and trends in incidence rates with time. In addition age and stage at presentation, gender and racial differences and finally prognosis and survival were collected when reported.
\end{abstract}

(c) 2013 Elsevier Ltd. All rights reserved.

\footnotetext{
* Corresponding author. Tel.: +972 86244256; fax: +972 86383652.

E-mail addresses: meravfra@gmail.com, meravfr@bgu.ac.il (M. Fraenkel), afaggian@unina.it (A. Faggiano), g.d.valk@umcutrecht.nl (G.D. Valk).

15 East 98th Street, 11th Floor, New York, NY 10029, USA. Tel.: +1 212241 7535; fax: +1 2122412276.

2 Tel.: +39 817464737; fax +39 815465443.

3 Tel.: +31 88 7556304; fax: +31887555514.
} 


\section{Introduction}

Gastroenteropancreatic neuroendocrine tumours (GEP-NETs) originate from diffuse neuroendocrine cells that are dispersed throughout the gastrointestinal tract and islets of Langerhans in the pancreas. These cells are unique in their capability to synthesize and secrete neuropeptides and hormones which have a role in normal physiology of the gastrointestinal tract (GIT) [1]. Histologically they may be identified by specific neuroendocrine markers such as synaptophysin and chromogranin A [2]. GEP-NETs are a heterogeneous group of tumours causing unique clinical syndromes and/or local symptoms, but may also be asymptomatic and discovered as an incidental finding [1].

Major changes have been made in the classification of NET tumours from their original identification by Oberndorfer in the early 1900's, who coined the name 'Karzinoide' to describe submucosal tumours in the small bowel which followed an indolent course when compared to adenocarcinoma [3]. The WHO classification of GEP-NET is based on histologic grade (based on the Ki-67 index) and degree of differentiation [4]. This new terminology of NET has yet not been fully accepted around the world, with many clinicians still using the term carcinoid. Moreover, some publications still classify NET according to the embryonic source of the organ (e.g. midgut carcinoid to describe ileal NET) $[5,6]$. The WHO 2010 system renders all NETs as neoplasms with a malignant potential [7,8]. This is in contrast to the older paradigm assuming these are rare, slow growing tumours with an indolent behaviour. We now know that some GEP-NETs are indolent and slow-growing and therefore remain stable for many years while others are poorly differentiated and carry a rapid and devastating course [1].

Inconsistency of nomenclature and classification of NET is the major limitation in elucidating the precise epidemiology of GEP-NET. This is reflected in the International Classification of Disease for Oncology coding system (ICD-O) that used one of several names to describe the same tumour: apudoma/carcinoid tumour/enterchromaffin cell carcinoid and so on (Table 1) [9]. In addition to this, older cancer registries, included only 'malignant carcinoids' while 'benign' tumours were not included, while other registries reported both benign and malignant tumours. This has changed with time, with the new ICD-O 10 coding already including benign NETs, and may explain some of the rise in incidence of GEP-NET around the world [9-11]. There are few, more recent publications on the epidemiology of GEP-NET that used the 2000 WHO classification, only one of which reports incidence rates [12-17]. Some publications from national, regional and institutional cancer registries or registries dedicated to NET patients, did not clearly define which classification was used for inclusion, and/or relied on the diagnosis given by the treating physician/institution [5,18-22].

A large autopsy series from Sweden reported an annual incidence rate of 8.4/100,000 for various NET (carcinoid) between the years 1958 and 1969 [23], which is much higher than what has been

Table 1

ICD oncology codes used for NET in the SEER registry. Copied with permission from Lawrence B. et al, Endocrinol Metab Clin N Am 2011:1-18.

\begin{tabular}{ll}
\hline NET histology & ICD code \\
\hline Carcinoid tumour & $8240 / 3$ \\
Enterochromaffin cell carcinoid & $8241 / 3$ \\
Goblet cell carcinoid & $8243 / 3$ \\
Composite carcinoid & $8244 / 3$ \\
Adenocarcinoid & $8245 / 3$ \\
Neuroendocrine carcinoma & $8246 / 3$ \\
Apudoma & $8248 / 3$ \\
Atypical carcinoid tumour & $8249 / 3$ \\
Stromal carcinoid & $9091 / 3$ \\
Islet cell carcinoma & $8150 / 3$ \\
Insulinoma & $8151 / 3$ \\
Glucagonoma & $8152 / 3$ \\
Gastrinoma & $8153 / 3$ \\
Mixed islet cell/exocrine adenocarcinoma & $8154 / 3$ \\
VIPoma & $8155 / 3$ \\
Somatostatinoma & $8156 / 3$ \\
\hline
\end{tabular}


reported in clinical series from those years $[9,24,25]$. This discrepancy points to the fact that many NET may remain asymptomatic throughout life and have no clinical significance. With the rising use of various imaging techniques for different indications there is also a rise in 'incidental NET' [26].

\section{Sources of information on GEP-NET epidemiology}

\section{National registries from the US and Europe}

The most informative sources for assessing GEP-NET epidemiology are from few national population based cancer registries from which the Surveillance Epidemiology and End Results (SEER) database in the US is the largest and most well known [9,27]. The End Results Group (ERG) from 1950 to 1969 and the Third National Cancer Survey (TNCS) between 1969 and 1971 preceded the SEER database and included early data on the epidemiology of GEP-NET in the US [24]. This was followed by the National Cancer Institute (NCI) SEER cancer registry. The SEER program was initiated in 1973, and was a composite of five states and four cities. Since then, $\mathrm{NCI}$ has added other states and regions in efforts to expand the registry of which the latest is the SEER 17 , created in 2000 , to now represent approximately $15 \%$ of the population of the United States and includes information on 5,553,822 cancer patients diagnosed from 1973 to 2007 (Fig. 1). Among these tumours 49,012 were NET and more than half of these were GEP-NET [9]. From these databases age adjusted annual incidence rates (IR) of the different GEP-NET have been assessed and published numerous times. Of note, up to 1986 'carcinoid' tumours were reported to the SEER database only if they were considered malignant.

Other national cancer registries reporting GEP-NET incidence originate from Norway, Sweden, Ireland, Netherlands, Denmark, Scotland and the United Kingdom [10,11,20,21,28-31]. Some of these national registries report incidence for all NET (including bronchial) $[29,30]$, or for all 'carcinoids' $[20,21,31]$, while others report on incidence by anatomic site $[10,11,28]$. National registries dedicated to NET patients that are not population based, like those from Germany, France, Spain and recently from Italy are an important source of data on the epidemiology of GEP-NET, but unfortunately are unable to report on incidence rates of these tumours [13,16,19,22].

Niedrele and colleagues prospectively collected all pathology reports using a standardized histopathological protocol based on the WHO 2000 classification of NET, from all pathology institutions in Austria between May 2004 and April 2005. Annual incidence rates of GEP-NET at the various anatomic sites were calculated (Fig. 2 and Table 2). This national report may be the most precise in reporting the true incidence of these tumours as we perceive them today [14].

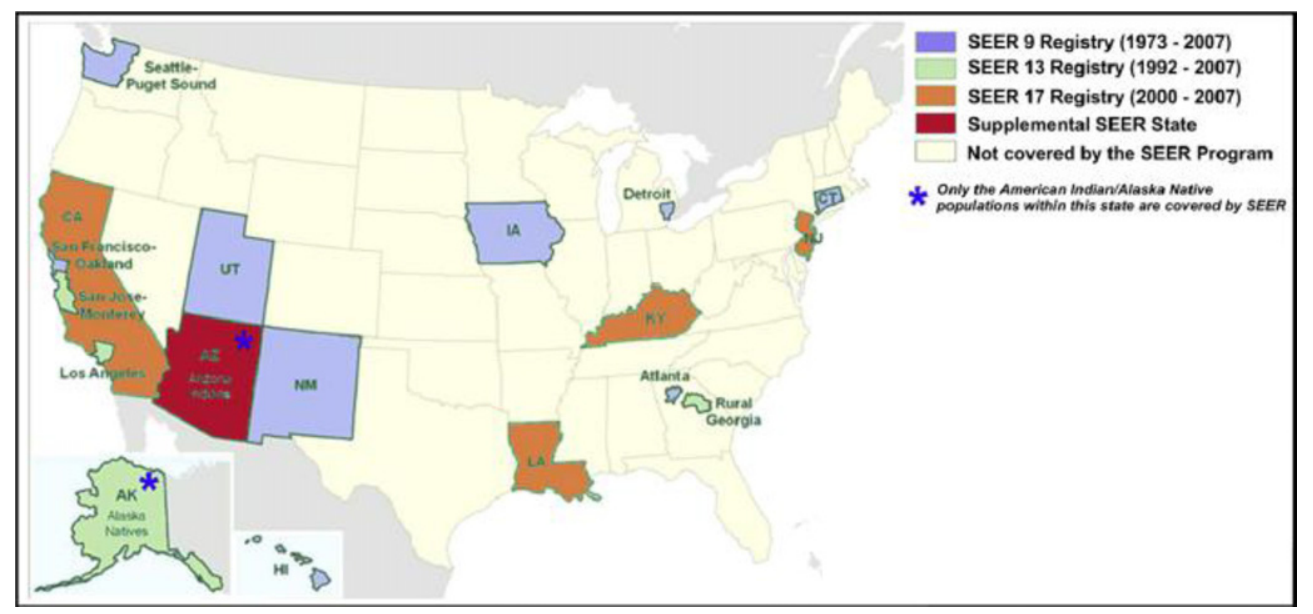

Fig. 1. Geographic distribution of the SEER registries. All shaded areas are covered by SEER 17 registry. Modified with permission from Lawrence B. et al, Endocrinol Metab Clin N Am 2011:1-18. 


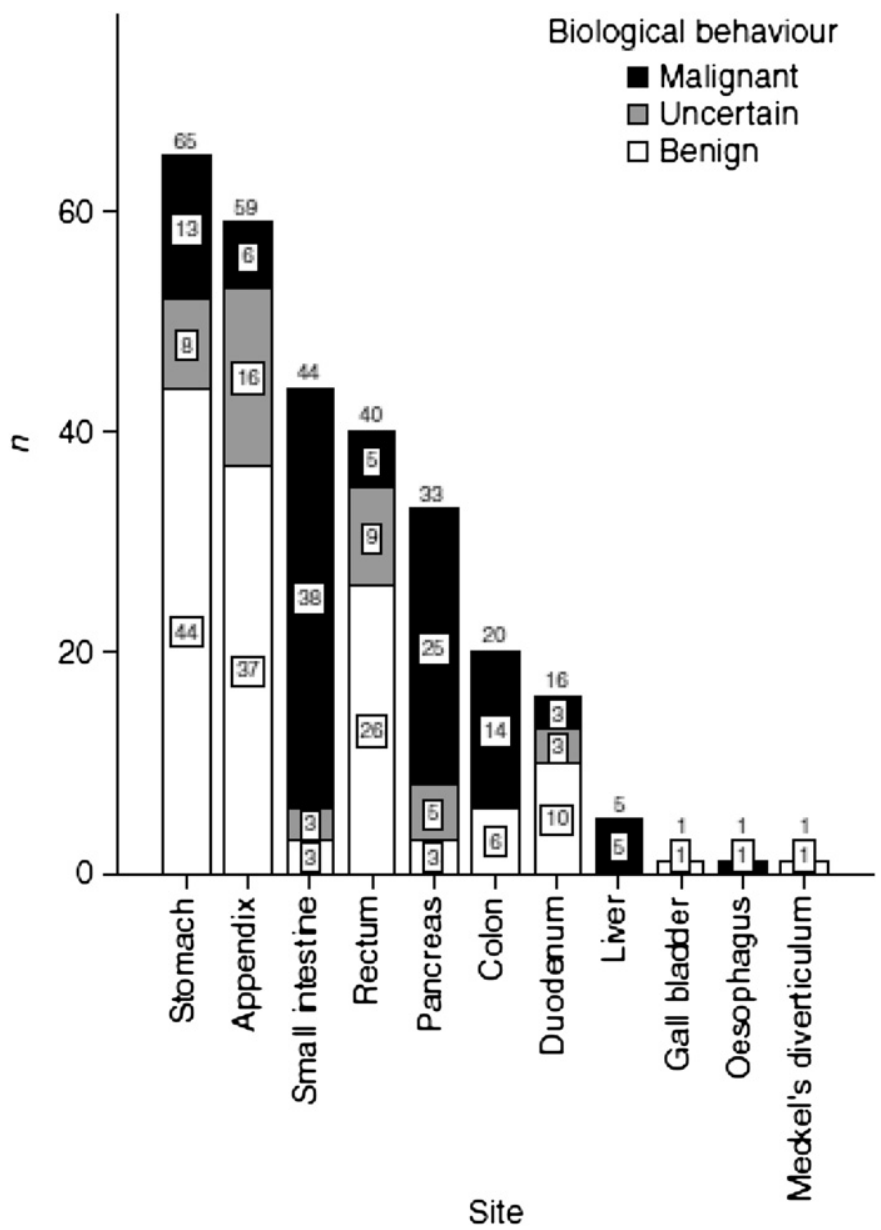

Fig. 2. Number, location and biologic behaviour of GEP NET in Austria 2004-2005 according to anatomic site. Adopted with permission from Niedrele et al., Endocrine-Related Cancer 2010:909-918.

\section{Table 2}

Site and incidence (per 100,000 population) of malignant GEP NETS in Austria. Adopted with permission from Niedrele et al, Endocrine-Related Cancer 2010:909918.

\begin{tabular}{ll}
\hline Site & Malignant NETs $^{\mathrm{a}}$ \\
\hline Oesophagus & 0.01 \\
Stomach & 0.08 \\
Duodenum & 0.02 \\
Pancreas & 0.19 \\
Small intestine excl. duodenum & 0.29 \\
Appendix & 0.08 \\
Colon (incl. rectosigmoid junction) & 0.06 \\
Rectum & 0.03 \\
Liver & 0.05 \\
Meckel's diverticulum & 0.00 \\
Gall bladder incl. bile duct & 0.00 \\
Total & 0.81 \\
\hline
\end{tabular}

a Study period: 05/2004-04/2005. 
Regional registries

Important epidemiology data has been published by regional registries in Europe and in the USA, including: Vaud in Switzerland, Tuscany in Italy, Burgundy in France, Girona in Spain and different regions within the US (Olmstead county MN, Utah, Florida) [6,32-41].

Registries outside of Europe and US

Reports on the epidemiology of GEP-NET outside the US and Europe are quite rare.

Younes et al reported the experience of a group of specialists from Brazilian institutions dedicated to the treatment of NET patients, in treating 1000 patients with various NETs. They reported on the relative frequency of the various primary sites and data on survival, but were unable to assess incidence or prevalence rates [18].

Ito et al reported incidence and prevalence rates of GEP-NETs from Japan in the year 2005 [5]. In this report GEP-NETs were reported according to embryonic origin, using the old classification of NET: foregut, midgut and hindgut and not using the WHO classification. Hindgut tumours were the most common of all GEP-NET in the Japanese population [5].

A retrospective study covering NET registration in one hospital in Taipei, Taiwan sheds light on the relative frequency of various NET in Taiwan between 1970 and 2005, with no population based incidence rates [15].

In 2011 Lim and colleagues published a retrospective study reviewing the pathology of all 470 GEPNETs diagnosed in Seoul Korea between the years 2001 and 2006, classified according to the 2000 WHO classification [17]. This was followed by a second report from Korea published in 2012 by the gastrointestinal pathology study group of Korean society of pathologists. In this comprehensive nationwide study, they collected information on 4951 pathology reports of GEP-NETs from 29 hospitals in Korea between 2000 and 2009, including distribution and survival analysis [12]. Both reports were not population based and therefore were unable to report annual incidence rates.

For clarity and simplicity all incidence rates (IR) reported in this review are of number of new cases per 100,000 population per year.

\section{Stomach}

NETs of the stomach have been traditionally classified as type I, associated with low acid of atrophic gastritis, type II associated with Zollinger Ellison syndrome in Multiple Endocrine Neoplasia type 1 (MEN1), type III 'sporadic' with a high malignant potential and type IV which is a poorly differentiated tumour (for review see reference [42]) [42,43]. Studies reporting on the epidemiology of Gastric NET (GNET) usually do not report on IR of the different subtypes, nevertheless it is accepted that type I are the most common, comprising $70-80 \%$ of cases followed by type III $14-20 \%$, while the other two types are much less common [42]. Registration of GNET in different cancer registries is variable, with some only documenting malignant tumours while others documenting benign and malignant tumours. In the SEER database up to 1986 only malignant GNETs were registered.

The relative frequency of GNET among all GEP-NET is in the range of 5-14.6\%, and has been rising according to the SEER database $[9,10,12,13,17,24,41,44-47]$. A recent report from Austria, found GNETs to be the most common of all GEP-NETs, the vast majority were benign (Fig. 2) [14], and in Korea they were the second most common location for GEP-NET after the rectum [12]. The earliest reports on IR of GNETs from the 1970's in the UK and the US are in the range of 0.01-0.03 but higher in Vaud Switzerland 0.07 [10,24,32,44,45] (Fig. 3A-C). Other countries in Europe reported higher IR in the 1990's IR $0.1-0.15[11,28,33]$. With time, there is a clear increase in IR of in the range of 11-16 fold within four decades in the US and UK, and a more mild increase over shorter time periods in Norway and Switzerland [9-11,27,32] (Fig. 3A-C). The rise in IR of GNETs represents to some extent a true rise in incidence, but has also been attributed to changes in registration and classification of GNETs, increased use of endoscopy, raise in awareness and of histologic diagnosis [9-11]. The rise in IR of GNETs parallels the rising prescription of proton pump inhibitors, but causality between the two has not been proven yet $[48,49]$. 

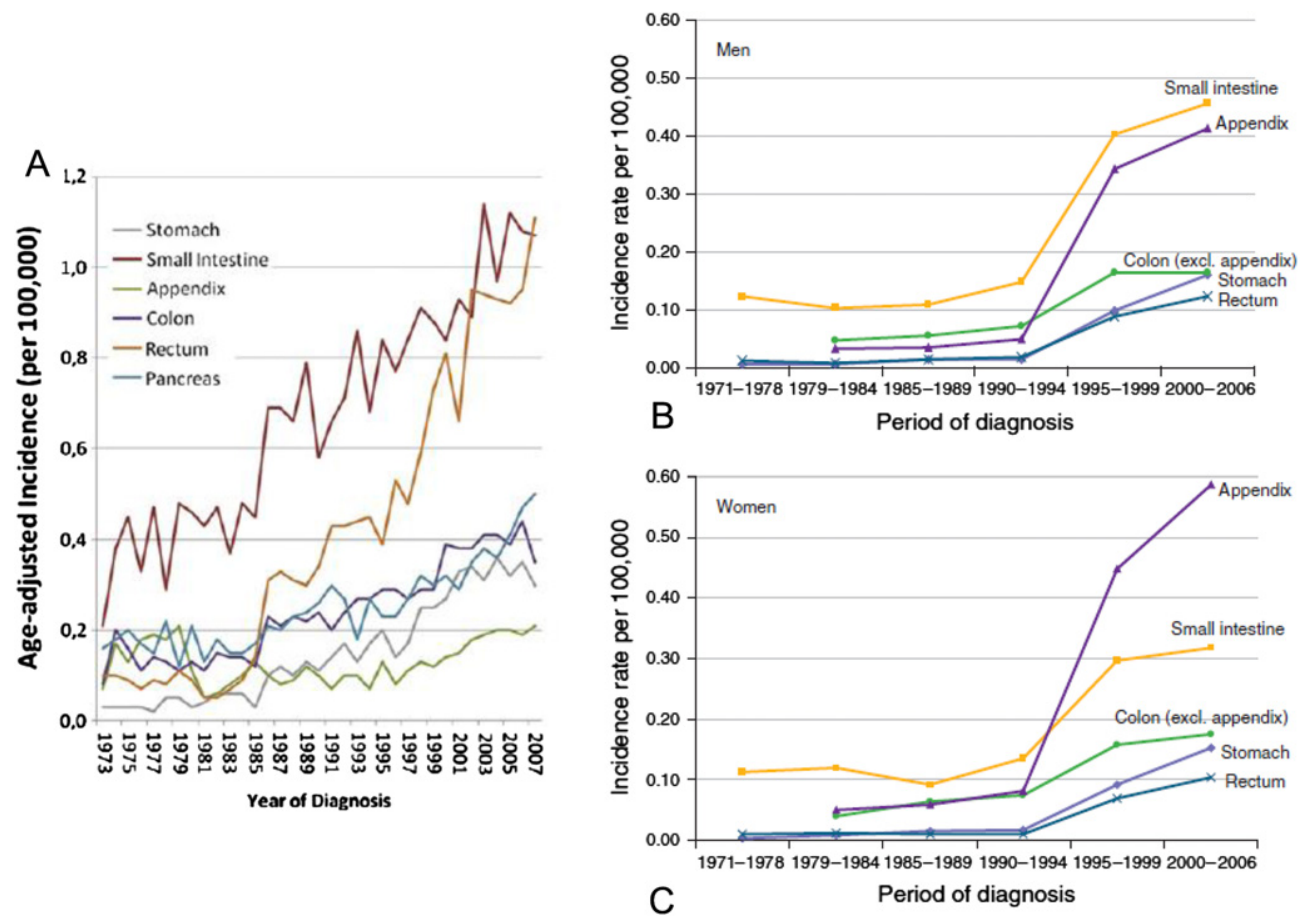

Fig. 3. Incidence rates of GEP NET in the US SEER database (A), males in UK (B) and females in UK (C). Modified with permission from Lawrence B. et al, Endocrinol Metab Clin N Am 2011: 1-18 (A) and from Ellis et al, Am J Gastroenterol 2010; 105:2563-2569 $(\mathrm{B}+\mathrm{C})$.

There are no clear cut gender differences in IR of GNETs [13,16,27,28,32,33,50]. In the US, IR of GNETs is highest in the black population [11,24,25,27,45,47,51]. Mean age at presentation of GNETs in the US and Europe is 60-64 years, and slightly older in Taiwan: 67 years [15,16,27,28,44,50], most patients presenting with local disease $[13,27,44,45]$. In the US, five-year survival rates of GNETs improved with time between 1973 and 2007 to reach a rate of 64\% [9,11,45] (Fig. 4). It has been speculated whether this rather low survival rate is negatively influenced by the inclusion of a larger proportion of malignant versus benign GNETs in the SEER database [9]. Others reported higher five-year survival rate based on the SEER database $75-82 \%$ [44,50]. Outside the US, five-year survival rates were reported: $45 \%$ between 1993 and 2004 in Norway, 61\% in Spain, 63.5\% in Tuscany, Italy (1985-2005) and 100\% in Taiwan (19702005) $[11,13,15,33]$. Five-year survival of malignant GNETs from 12 European countries between 1985 and 1994 was low at 45.6\%, with prognosis being the best in Northern European countries [52]. This wide variation in survival of patients with GNETs may be due to geographic changes in classification, diagnosis and management.

\section{Small intestine}

Of all small intestinal (SI) NETs including the duodenum, jejunum and ileum; the ileum is the most common $[9,10,53]$. SI NETs have been the most common among GEP-NETs in the US and Europe, comprising 39\% of malignant GEP-NETs in UK between 2000 and 2006, and $18.6 \%$ of all NET in the pan SEER registry between 1973 and 2007 [9,10]. In the Netherlands and Germany SI NETs were the second most common GEP-NETs [19,29]. Interestingly, in eastern Asia SI NET are much less common than in Western countries $[5,12,15,17]$.

Annual age adjusted IR of small intestinal NETs is available from multiple countries in Europe $[10,11,14,28,32-35,39,41,54]$ and has been published multiple times from the US SEER database 


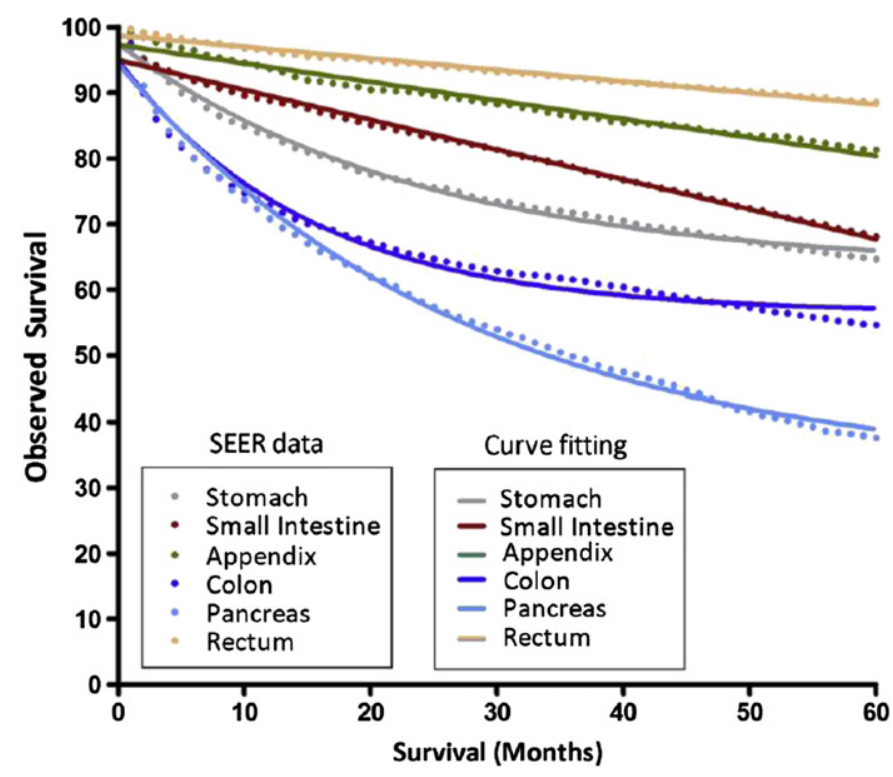

Fig. 4. Observed five-year survival rates for GEP-NET primary sites in SEER 17 (1973-2007). Modified with permission from Lawrence B. et al, Endocrinol Metab Clin N Am 2011: 1-18.

$[9,24,27,44,47,50,53,55,56]$, encompassing various periods between the years1960-2008. In many countries IR of SI NET are the highest among all GEP-NETs [6,9-11,14,22,30,32,34,35,44]. In Japan midgut carcinoids had the lowest IR compared to hindgut and foregut carcinoid $(0.24,0.64$ and 1.26 respectively) [5]. Incidence rates were the lowest in the 1970's between 0.07 and 0.48 (minimum in Burgundy France and maximum in the USA) [9,47,54]. With time, there is clear increase in the IR of SI NET: 3.8 and 1.8 fold in men and women respectively in the UK between 1971 and 2006 (Fig. 3B+C) [10] More modest changes were observed in Sweden [28], Burgundy France, Vaud Switzerland and Norway [11,28,32,35]. The US demonstrated a nearly three fold increase in IR from 1973 to 2007 (0.38-1.08, respectively) [9,50] (Fig. 3). Despite the similar range in fold increase in IR of SI NET in the US and UK with time, incidence rates in the US are more than twice as high as those in the UK during the same years (2000-2006): 1.08 in the US SEER database and only 0.46 and 0.32 in males and females in the UK respectively (Fig. 3A-C) [9,10]. IR rates in Norway (2000-2004) were similar to those in the US 1.01 [9,11], with lower incidence reported over similar time frames in Austria 0.29 (Table 2) [14].

IR of SI NET increases with age starting at age 40, reaching a peak at the eighth decade of life $[9,10,28,29]$. Mean age at diagnosis is between 59 and 65 years $[15,19,27,28,44,50]$, most $(58-71 \%)$ patients presenting with regional or distant spread $[14,27,47,53]$. SI NETs are more common in males $[10,11,28,32,33,47,54]$, and the US SEER database points at strong racial differences, with incidence rates that are higher in African Americans compared to whites (both males and females) [11,27,47,53]. Godwin reported five-year survival rate of SI NET to be 52\% in the ERG and TNCS US databases [24]. Five-year survival rates in the US have increased since, but data from the US SEER database showed no improvement of survival between 1973 and 2007, in the range of 62-71\% (Fig. 4) [9,53,55]. Higher five-year survival rates were reported by Maggard: $75 \%$ in the same SEER population and by Tsikitis et al who reported as of 2008 , five-year survival rates of $86.2 \%$ with improvement with time in nonHispanic whites only [50]. Five-year survival rates in Europe were low in Norway and France 59 and $56 \%$ respectively and higher in Italy and Spain: 72-89\% [11,13,33,54]. Members of the EUROCARE working group found five-year survival rates of malignant SI NET in the range of 55-64\% among 19 centres in 12 European countries, the lowest in Eastern and the highest in Western European countries [52]. 


\section{Pancreatic neuroendocrine tumours}

Data on the epidemiology of pancreatic neuroendocrine tumours (pNET) are limited compared to other GEP-NETs. These are rare tumours with an estimated incidence rate in several population studies of $<1$ [57]. In autopsy studies pNET occur at a higher rate with percentages of patients with a PNET ranging from 0.07 up to 10\% [57]. This wide range is explained by improved awareness and well defined histological and immunohistochemical criteria that allow clinicians to reliably identify PNET and differentiate them from other types of tumours [46]. Of consequence, a number of PNETs is now diagnosed on surgical samples or biopsy, even when there is no functioning endocrine syndrome or local symptoms suggesting this type of tumour. Furthermore, high sensitive and specific imaging techniques, such as computed tomography, SPECT with ${ }^{111}$ In-Pentetreotide and Positron Emission Tomography (PET) with ${ }^{68} \mathrm{Ga}$-DOTATATE, ${ }^{11} \mathrm{C}$ 5-HTP and ${ }^{18} \mathrm{~F}$-DOPA, multidetector-row CT and endoscopic ultrasound are now available helping to detect and localize symptomatic and asymptomatic PNETs $[26,58,59]$.

In NET registries from Spain, Italy and Germany PNETs were the most common among GEP-NET $[13,16,19]$, while in France, Girona province in Spain and in a single institution in Seoul, Korea they were the second most common [17,22,41]. In the pan-SEER population database between 1973 and 2007, PNET consisted only 7.34\% of all NET, not changing dramatically in this time frame [9].

There are few reports from European registries indicating that the incidence of PNET is low, but rising over the last decades. Older studies reported an IR of approximately 0.1 whereas more recent studies from Europe reported an incidence as high as 0.3 [11,14,33,35,41]. Incidence rates of PNET from the SEER database have been reported several times [9,11,27,60,61], showing, similar to what has been shown in Europe, a rise in incidence ranging from 0.17 in the 1970's compared to 0.43 in the most recent report (2003-2007) (Fig. 3A) [9]. Much higher IR of PNET was reported in Japan: 1.01 [5].

Peak incidence rates of PNET are in the sixth to eighth decades and median age at presentation is 60 years [16,19,27,29,60,61]. PNETs are slightly more common in males $[11,27,35,60,61]$ and in the US a slightly higher IR in African Americans compared to whites has been reported [11,27,61]. The majority of patients with PNET (77-86\%) have regional or distant spread at presentation [13,14,27,29,60,61]. Patients with the multiple endocrine neoplasia Type 1 (MEN1) have a chance of 35-75\% to develop a clinical manifest PNET. In the course of this syndrome PNETs occur at a younger age, are more often multiple and are, in addition, diagnosed at an earlier stage because of the periodic screening of mutation carriers [62].The familial von Hippel-Lindau disease is also associated with a higher pNET incidence but not as high as MEN1 [63,64].

PNETs are classified according to the cell of origin. Functioning PNETs secrete bioactive compounds leading to a clinical syndrome such as insulinomas, gastrinomas and rarer syndromes such as VIPomas, somatostatinomas and glucagonomas while others are considered non-functional. Not all hormonal secretion leads to clinical syndromes. Non-functioning tumors are more common than functioning PNETs [61]. From several studies it has been estimated that of the functioning PNETs, insulinomas are the most frequently occurring, followed by gastrinomas as the second most commonly occurring functional tumour type. VIPoma and glucagonomas are more rare [57].

Based on data from the SEER database it seems that pancreatic NETs exhibited the lowest five-year survival (27-37.6\%) when compared with other GEP-NETs, survival being the lowest in patients presenting at advanced stage (Fig. 4) [9,11,27,60,61], and better in patients with functional PNETs [56]. Higher five-year survival rates have been reported from Norway (43\%) [11] Spain (71-78\%) [13], a regional registry in Burgundy France (44\%) and from Tuscany Italy (62\%) [33]. Consistent with these reports the EUROCARE study group found five-year survival rates of patients diagnosed with malignant PNET to be higher in Northern Europe (49\%) compared to Eastern European countries (8.5\%) [52]. Due to the heterogeneity of the cohorts of patients with PNET, it is difficult to draw conclusions about functional status as prognostic factor in terms of survival [57].

\section{Colorectal NET}

Describing the epidemiology of NET of the colon is complicated by the fact that different series have described these tumours either separately or included them with the rectal NET, termed together as 
'colorectal NET'. In addition some have included appendiceal NET as part of colon NET [33]; in this review NET of the appendix will be discussed separately from colon NET.

In France, overall colorectal NET IR was 0.19 in men and 0.12 in women between 1976 and 1999 [35]. Reports from Vaud Switzerland from a similar time period (1974-1997) showed much higher IR, with a strong female predominance: 0.7 in males and 1.74 in females. This registry included appendiceal NET in the group of colorectal NET, which may explain the higher incidence and the gender differences. In this time frame, incidence remained high without change in Swiss women, while rates more than doubled in Swiss men from 0.45 to 0.98 [32]. In Japan IR of hindgut carcinoid, which includes colon and rectum, was the highest compared to other GEP-NETS, with an IR of 1.26 in the year 2005 [5]. Five-year survival rate of colorectal NET from 12 European countries between 1975 and 2000 was in the range of 49.9-67\%, lowest in Eastern and highest in Western European countries [52].

\section{Colon NETs (rectum excluded)}

NETs have been documented in all parts of the colon: cecum, ascending, transverse, descending and sigmoid. Within the colon there is a predominance of right sided NET $[9,10,27,40,47,55,65]$.

Incidence rates for Colon NET (CNET) have been reported over different time periods in Europe and the US. IR from the 1970's in the UK was 0.04 and 0.05 in females and males respectively [10] (Fig. 3B+C), while in Austria NET registration within one year between 2004 and 2005 showed similarly low incidence of malignant colon NET 0.06 (Table 2) [14]. Higher incidence rates were reported between 1983 and 1998 in Sweden at 0.1 [28], and in Norway between 1993 and 1997 at 0.19 [11]. The earliest reports on incidence of CNET from the US, as reported to the ERG and TCNS, were 0.07-0.15 (lowest in white females and highest in black males) [24]. Multiple reports of CNETs from the SEER database show low incidence rates in the mid 70's in the range of 0.08-0.15 [9,44,47,55]. With time, IR of colon NETs has increased only mildly in the UK (Fig. 3B+C) and Norway [10,11]. While IR more than doubled in the SEER database between 1973 and 2007: from 0.15 to 0.4 (Fig. 3A) [9,50]. This increase in incidence of NET of the colon around the world has been attributed among others, to the increased use of screening colonoscopy and other imaging modalities, which discover these tumours in asymptomatic patients $[9,10,50]$.

Data on gender differences in IR of CNET are conflicting [13,19,28,32,33,35,40,47,50]. Reports from the US SEER database show higher IR in African Americans compared to whites, while rates were higher in whites in the Connecticut tumour registry [11,24,27,40,47,50].

Incidence of CNETs increases with age starting at age 40-50, reaching a peak in the seventh to ninth decade $[9,28,40,50]$. Mean age of patients with colon NET is reported as 63-68 in the US [27,50], and in the range of 54-61 in European series [19,28,29], most patients (53-86\%) presenting with either regional or distant spread $[13,24,27,40,44,50]$.

Five-year survival rates of CNET in Europe have been reported from Norway: 41\% (1993-2004), Spain: 65\% (2001-2008) and Burgundy France: 36\% (1976-1999) [11,13,33,35]. Early reports from the ERG/TCNS US data reported five-year survival rates of 52\% between 1950 and 1971 [24]. Gustafsson reported on an increase in five-year survival from 44\% in 1973 to 53.6\% in 2004 [55], higher rates of five-year survival were reported by Maggard: 69\% between the years 1973 and 1997 [44], and by Crocetti 72-87\% between 1992 and 1999 [65]. Modlin et al reported no improvement in five-year survival between two time periods: 1973-1991 and 1992-1999 in the range of 60\%, which remain the same in the SEER 17 encompassing the years 1973-2007 (Fig. 4) [9,47]. In contrast to this, the latest report on GEP-NETs from the US SEER database claimed that there was an increase in five-year survival rates of patients with NET of the colon, in the years 1973-2008, reaching an average of 67\% [50]. These conflicting five-year survival rates in the SEER database require further explanation.

\section{Rectal NETs}

In the SEER database the frequency of rectal NET (RNET) has been increasing steadily and as of 2000-2007, RNETs are slightly more common than small intestinal NET, each comprising about $17 \%$ of all NET $[9,27,50]$. In Taiwan during 35 years of registration, rectal NETs were the most common of all NET (61\%) and in two reports from Korea they were the most common of GEP-NET (48-55\%) $[12,15,17]$. In Europe RNETs are much less common within all GEP-NET, 9\% in the UK and 5.8\% in Spain $[10,13]$. 
IR of RNETs was as low as 0.01 in the UK between 1970 and 1989 [10], but higher in Italy, Norway and Sweden when reported from the mid 1980's and on 0.1-0.22 [11,28,33,34]. Notably IR of malignant RNET in Austria between 2004 and 2005 was 0.08 (Table 2) [14]. IR in the US from the 1970's was reported several times, and was in the range of 0.09-0.4 [24,44,47]. IR of rectal NET has been rising more than ten fold in the UK [10] (Fig. 3B+C), with a much less dramatic increase in Norway (15\%) [11]. In the US SEER population IR of RNETs have increased ten fold between 1973 and 2007 (Fig. 3A) $[9,27,50]$. IR of RNET in the US SEER database between 2000 and 2006 is about ten times as high as the IR in the same years in the UK (Fig. 3A-C). Not all increase in IR can be attributed to higher rates of colonoscopy and sigmoidoscopy $[9,10]$.

Mean age at diagnosis of rectal NET is between age 52-57 [15,19,27,28,44], and most patients present with local disease [13,24,27,44,47]. RNETs are more common in males in some but not all populations [11,13,16,27,47], and in the US these tumours are far more common in black males and females compared to whites [11,24,27,47,51]. In the US, RNETs have an excellent five-year survival reaching about $90 \%$ (Fig. 4) $[9,11,24,44,47,50]$. Similarly high five-year survival rates have been reported from Taiwan: $86 \%$, with lower rates in Norway and Spain $74 \%$ and $64 \%$ respectively $[11,13,15]$.

\section{Appendix}

Reporting of malignant and benign appendiceal NET varied with time in different countries. Furthermore, in some countries NET of the appendix was included as part of all colon NET, a classification that letter was changed. Goblet cell tumour of the appendix was inconsistently included or excluded as a NET in different registries, profoundly affecting five-year survival rates of this group on NETs.

NET of the appendix (Appendiceal NET; ANET) is considered one of the least common in the SEER database (3.44\% of all NETS 1973-2007) [9,27,50] in Norway (4.8\% of all NET 1993-2004) [11] and in the far east [12,15,17]. Having said that, in the ERG (1950-1969) and TCNS (1969-1971) US databases, which preceded the SEER, appendiceal NET were the most common: 43.9\% and 35\% of all NET. This 'drop' in frequency of NET of the appendix is due to the fact that the first two registries included both benign and malignant ANET, while the SEER up to 1986 reported only malignant tumours [51]. In several European countries the relative frequency of ANET is among the highest; comprising $38 \%$ of all GI NET in the UK (2000-2006) and of GEP-NET in Girona, Spain (1994-2004) [10,14,21,28-30,33,34,41]. Part of this geographical variability is attributed to differences in registration.

Early reports on IR of appendiceal ANET from Europe, showed a rate as low as 0.03 in the UK in the early 1970s' [10] with higher rates in the 1980-1990s' in Tuscany Italy, Norway and Sweden 0.09, 0.1 and 0.4 respectively $[11,28,34]$. Incidence rate of malignant NET of the appendix in Austria was 0.08 between 2004 and 2005 (Table 2) [14]. In the US TNCS database IR were as high as 0.79 in white females between 1969 and 1971 [24]. Early SEER data reported lower overall rates between 0.07 and 0.15 $[9,44,47,55]$. These differences are again explained by inclusion of only malignant ANETs in the SEER database [51].

The incidence rate of ANET increased dramatically with time in the UK: an 11 fold increase in males and ten fold increases in females, largely explained by the inclusion of benign tumours in cancer registration in recent years. Having said that, there was also a five fold increase in the rates of malignant tumours in this site [10]. Milder increases in IR were also observed in Sweden and Norway [28]. Analysis of the SEER data shows an early decrease and then a stabilization of IR [11]. The initial decrease in IR has been attributed to differences in registration of all vs. only malignant NETs $[9,11,27,47,50]$.

Appendiceal NET is most commonly an incidental finding on appendectomy preformed for acute appendicitis and this partially explains the younger age at presentation of this subset of GEP-NET ranging between age 31 and 49 in different series [47]. This may also explain the very low rate of regional or distant metastasis seen with these tumours [16,19,27-29,47,50].

IR of ANET is twice as high in females compared to males in Europe and in the US [13,27,29]. These gender differences have been attributed by some to the higher rates of appendectomies and gynaecological procedures in females. In the US incidence rates by race varied with time with no consistent racial differences between blacks and whites [10,11,24,27-29,44,47,50].

Survival rates of appendiceal NET are higher than most GEP-NET both in Europe and the US. In Europe five-year survival rates of $74-95 \%$ were reported, being the lowest in Norway where inclusion 
of Goblet cell tumours lowered five-year survival rates significantly [11,24,27,47]. In the US, five-year survival rates between 1973 and 2008 were reported as high as $90 \%$ by Tsikitis and colleagues, but only $81 \%$ by Lawrence et al $[11,13,29]$. Higher survival rates were reported in series from the 1970 's when benign tumours comprised the majority of NET at this site $[9,50]$. This relatively high five-year survival rate is explained among others, by the specific characteristics of this anatomic location but more probably by the incidental discovery and curative surgery done early for these tumors [24,55].

\section{Summary}

Gastro-entero-pancreatic neuroendocrine tumors (GEP-NETs) are a heterogeneous group, formally called 'carcinoids'. They can involve any part of the gut with a wide range of malignant potential. Reports on the epidemiology of GEP-NET from around the world are not homogenous in the type of tumors included, classification and terminology, with the new WHO classification yet to gain a more worldwide acceptance. Data on the epidemiology of GEP-NET from many parts of the world is still lacking.

The relative frequency and annual incidence rate per 100,000 population of GEP-NETs change with time and with the geographical location around the world. NET of the rectum is the most common in east Asia and in recent years also in the US SEER database. Small intestinal NETs are still the most common in males in the UK while appendiceal NETs are more common in females. Registries that are dedicated to NET patients show a much higher frequency of pancreatic NET when compared to population based studies. Incidence rates of GEP-NETs have been increasing with time with the largest increases found in NET of the stomach and rectum. Incidence rates of GPE NETs show gender and racial differences which varies with tumour localization. Prognosis of GEP-NET patients is high when compared to adenocarcinoma of the same location. Five-year survival rates are highest in rectal and appendiceal NET, but lower in small intestinal and pancreatic NETs, with large variability in survival between countries in Europe and the US.

\section{Practice points}

- Annual incidence rates of most gastro-entero-pancreatic tumors are rising around the world in recent years.

- NET of the rectum is the most common GEP-NET in the Far East and now also in the US.

- The rise in incidence of GEP-NET is only partially explained by increases in anatomic, functional and luminal imaging done for screening or as part of an investigation for other indications.

- The prevalence of GEP-NET is not reported in many parts of the world.

\section{Research agenda}

- Uniform classification, preferably using the updated WHO system should be accepted worldwide and used in future studies on the epidemiology of GEP-NET.

- National cancer and NET registries should collaborate in order to supply data on age adjusted annual incidence rates and prevalence of these tumors.

- Further research is needed to find the environmental and genetic factors affecting differences in the epidemiology of GEP-NET around the world.

- Increased awareness and improved treatment modalities by multidisciplinary teams are expected to raise five-year survival rates of various GEP-NETs. 


\section{References}

[1] Modlin IM, Oberg K, Chung DC, Jensen RT, de Herder WW, Thakker RV, et al. Gastroenteropancreatic neuroendocrine tumours. Lancet Oncol 2008;9(1):61-72.

[2] Klöppel G, Couvelard A, Perren A, Komminoth P, McNicol AM, Nilsson O, et al. ENETS Consensus Guidelines for the Standards of Care in Neuroendocrine Tumors: towards a standardized approach to the diagnosis of gastroenteropancreatic neuroendocrine tumors and their prognostic stratification. Neuroendocrinology 2009;90(2):162-6.

[3] Modlin IM, Shapiro MD, Kidd M. Siegfried Oberndorfer: origins and perspectives of carcinoid tumors. Hum Pathol 2004; 35(12):1440-51.

[4] Kloppel G, Perren A, Heitz PU. The gastroenteropancreatic neuroendocrine cell system and its tumors: the WHO classification. Ann N Y Acad Sci 2004;1014:13-27.

[5] Ito T, Sasano H, Tanaka M, Osamura RY, Sasaki I, Kimura W, et al. Epidemiological study of gastroenteropancreatic neuroendocrine tumors in Japan. J Gastroenterol 2010;45(2):234-43.

[6] Perez EA, Koniaris LG, Snell SE, Gutierrez JC, Sumner 3rd WE, Lee DJ, et al. 7201 Carcinoids: increasing incidence overall and disproportionate mortality in the elderly. World J Surg 2007;31(5):1022-30.

[7] Klimstra DS, Modlin IR, Adsay NV, Chetty R, Deshpande V, Gönen M, et al. Pathology reporting of neuroendocrine tumors: application of the Delphic consensus process to the development of a minimum pathology data set. Am J Surg Pathol 2010; 34(3):300-13.

[8] Klimstra DS, Modlin IR, Coppola D, Lloyd RV, Suster S. The pathologic classification of neuroendocrine tumors: a review of nomenclature, grading, and staging systems. Pancreas 2010;39(6):707-12.

[9] Lawrence B, Gustafsson BI, Chan A, Svejda B, Kidd M, Modlin IM. The epidemiology of gastroenteropancreatic neuroendocrine tumors. Endocrinol Metab Clin North Am 2011;40(1):1-18 [vii].

[10] Ellis L, Shale MJ, Coleman MP. Carcinoid tumors of the gastrointestinal tract: trends in incidence in England since 1971. Am J Gastroenterol 2010;105(12):2563-9.

[11] Hauso O, Gustafsson BI, Kidd M, Waldum HL, Drozdov I, Chan AK, et al. Neuroendocrine tumor epidemiology: contrasting Norway and North America. Cancer 2008;113(10):2655-64.

[12] Cho MY, Kim JM, Sohn JH, Kim MJ, Kim KM, Kim WH, et al. Current trends of the incidence and pathological diagnosis of gastroenteropancreatic neuroendocrine tumors (GEP-NETs) in Korea 2000-2009: Multicenter Study. Cancer Res Treat 2012;44(3):157-65.

[13] Garcia-Carbonero R, Capdevila J, Crespo-Herrero G, Díaz-Pérez JA, Martínez Del Prado MP, Alonso Orduña V, et al. Incidence, patterns of care and prognostic factors for outcome of gastroenteropancreatic neuroendocrine tumors (GEP-NETs): results from the National Cancer Registry of Spain (RGETNE). Ann Oncol 2010;21(9):1794-803.

[14] Niederle MB, Hackl M, Kaserer K, Niederle B. Gastroenteropancreatic neuroendocrine tumours: the current incidence and staging based on the WHO and European Neuroendocrine Tumour Society classification: an analysis based on prospectively collected parameters. Endocr Relat Cancer 2010;17(4):909-18.

[15] Li AF, Hsu CY, Li A, Tai LC, Liang WY, Li WY, et al. A 35-year retrospective study of carcinoid tumors in Taiwan: differences in distribution with a high probability of associated second primary malignancies. Cancer 2008;112(2):274-83.

[16] Faggiano A, Ferolla P, Grimaldi F, Campana D, Manzoni M, Davì MV, et al. Natural history of gastro-entero-pancreatic and thoracic neuroendocrine tumors. Data from a large prospective and retrospective Italian Epidemiological study: the NET Management Study. J Endocrinol Invest 2012;35(9):817-23.

[17] Lim T, Lee J, Kim JJ, Lee JK, Lee KT, Kim YH, et al. Gastroenteropancreatic neuroendocrine tumors: incidence and treatment outcome in a single institution in Korea. Asia Pac J Clin Oncol 2011;7(3):293-9.

[18] Younes RN. Neuroendocrine tumors: a registry of 1,000 patients. Rev Assoc Med Bras 2008;54(4):305-7.

[19] Ploeckinger U, Kloeppel G, Wiedenmann B, Lohmann R. The German NET-registry: an audit on the diagnosis and therapy of neuroendocrine tumors. Neuroendocrinology 2009;90(4):349-63.

[20] Buchanan KD, Johnston CF, O'Hare MM, Ardill JE, Shaw C, Collins JS, et al. Neuroendocrine tumors. A European view. Am J Med 1986;81(6B):14-22.

[21] Watson RG, Johnston CF, O'Hare MM, Anderson JR, Wilson BG, Collins JS, et al. The frequency of gastrointestinal endocrine tumours in a well-defined population - Northern Ireland 1970-1985. Q J Med 1989;72(267):647-57.

[22] Lombard-Bohas C, Mitry E, O'Toole D, Louvet C, Pillon D, Cadiot G, et al. Thirteen-month registration of patients with gastroenteropancreatic endocrine tumours in France. Neuroendocrinology 2009;89(2):217-22.

[23] Berge T, Linell F. Carcinoid tumours. Frequency in a defined population during a 12-year period. Acta Pathol Microbiol Scand A 1976;84(4):322-30.

[24] Godwin 2nd JD. Carcinoid tumors. An analysis of 2,837 cases. Cancer 1975;36(2):560-9.

[25] Modlin IM, Sandor A, Tang LH, Kidd M, Zelterman D. A 40-year analysis of 265 gastric carcinoids. Am J Gastroenterol 1997; 92(4):633-8.

[26] Zarate X, Williams N, Herrera MF. Pancreatic incidentalomas. Best Pract Res Clin Endocrinol Metab 2012;26(1):97-103.

[27] Yao JC, Hassan M, Phan A, Dagohoy C, Leary C, Mares JE, et al. One hundred years after "carcinoid": epidemiology of and prognostic factors for neuroendocrine tumors in 35,825 cases in the United States. J Clin Oncol 2008;26(18):3063-72.

[28] Hemminki K, Li X. Incidence trends and risk factors of carcinoid tumors: a nationwide epidemiologic study from Sweden. Cancer 2001;92(8):2204-10.

[29] Quaedvlieg PF, Visser O, Lamers CB, Janssen-Heijen ML, Taal BG. Epidemiology and survival in patients with carcinoid disease in The Netherlands. An epidemiological study with 2391 patients. Ann Oncol 2001;12(9):1295-300.

[30] Westergaard T, Frisch M, Melbye M. Carcinoid tumors in Denmark 1978-1989 and the risk of subsequent cancers. A population-based study. Cancer 1995;76(1):106-9.

[31] Newton JN, Swerdlow AJ, dos Santos Silva IM, Vessey MP, Grahame-Smith DG, Primatesta P, et al. The epidemiology of carcinoid tumours in England and Scotland. Br J Cancer 1994;70(5):939-42.

[32] Levi F, Te VC, Randimbison L, Rindi G, La Vecchia C. Epidemiology of carcinoid neoplasms in Vaud, Switzerland, 1974-97. Br J Cancer 2000;83(7):952-5. 
[33] Caldarella A, Crocetti E, Paci E. Distribution, incidence, and prognosis in neuroendocrine tumors: a population based study from a cancer registry. Pathol Oncol Res 2011;17(3):759-63.

[34] Crocetti E, Buiatti E, Amorosi A. Epidemiology of carcinoid tumours in central Italy. Eur J Epidemiol 1997;13(3):357-9.

[35] Lepage C, Bouvier AM, Phelip JM, Hatem C, Vernet C, Faivre J. Incidence and management of malignant digestive endocrine tumours in a well defined French population. Gut 2004;53(4):549-53.

[36] Hodgson N, Koniaris LG, Livingstone AS, Franceschi D. Gastric carcinoids: a temporal increase with proton pump introduction. Surg Endosc 2005;19(12):1610-2.

[37] DiSario JA, Burt RW, Vargas H, McWhorter WP. Small bowel cancer: epidemiological and clinical characteristics from a population-based registry. Am J Gastroenterol 1994;89(5):699-701.

[38] Service FJ, McMahon MM, O'Brien PC, Ballard DJ. Functioning insulinoma-incidence, recurrence, and long-term survival of patients: a 60-year study. Mayo Clin Proc 1991;66(7):711-9.

[39] Levi F, Randimbison L, Franceschi S, La Vecchia C. Descriptive epidemiology of malignant carcinoids in the Swiss Canton of Vaud. Int J Cancer 1993;53(6):1036-7.

[40] Ballantyne GH, Savoca PE, Flannery JT, Ahlman MH, Modlin IM. Incidence and mortality of carcinoids of the colon. Data from the Connecticut Tumor Registry. Cancer 1992;69(10):2400-5.

[41] Alsina M, Marcos-Gragera R, Capdevila J, Buxó M, Ortiz RM, Barretina P, et al. Neuroendocrine tumors: a population-based study of incidence and survival in Girona Province, 1994-2004. Cancer Epidemiol 2011;35(6):e49-54.

[42] Scherübl H, Cadiot G, Jensen RT, Rösch T, Stölzel U, Klöppel G. Neuroendocrine tumors of the stomach (gastric carcinoids) are on the rise: small tumors, small problems? Endoscopy 2010;42(8):664-71.

[43] Ruszniewski P, Delle Fave G, Cadiot G, Komminoth P, Chung D, Kos-Kudla B, et al. Well-differentiated gastric tumors/ carcinomas. Neuroendocrinology 2006;84(3):158-64.

[44] Maggard MA, O'Connell JB, Ko CY. Updated population-based review of carcinoid tumors. Ann Surg 2004;240(1):117-22.

[45] Modlin IM, Lye KD, Kidd M. A 50-year analysis of 562 gastric carcinoids: small tumor or larger problem? Am J Gastroenterol 2004;99(1):23-32.

[46] Plöckinger U, Rindi G, Arnold R, Eriksson B, Krenning EP, de Herder WW, et al. Guidelines for the diagnosis and treatment of neuroendocrine gastrointestinal tumours. A consensus statement on behalf of the European Neuroendocrine Tumour Society (ENETS). Neuroendocrinology 2004;80(6):394-424.

[47] Modlin IM, Lye KD, Kidd M. A 5-decade analysis of 13,715 carcinoid tumors. Cancer 2003;97(4):934-59.

[48] Jensen RT. Consequences of long-term proton pump blockade: insights from studies of patients with gastrinomas. Basic Clin Pharmacol Toxicol 2006;98(1):4-19.

[49] Abraham NS. Proton pump inhibitors: potential adverse effects. Curr Opin Gastroenterol 2012;28(6):615-20.

[50] Tsikitis VL, Wertheim BC, Guerrero MA. Trends of incidence and survival of gastrointestinal neuroendocrine tumors in the United States: a seer analysis. J Cancer 2012;3:292-302.

[51] Modlin IM, Sandor A. An analysis of 8305 cases of carcinoid tumors. Cancer 1997;79(4):813-29.

[52] Lepage C, Ciccolallo L, De Angelis R, Bouvier AM, Faivre J, Gatta G. European disparities in malignant digestive endocrine tumours survival. Int J Cancer 2010;126(12):2928-34.

[53] Modlin IM, Champaneria MC, Chan AK, Kidd M. A three-decade analysis of 3,911 small intestinal neuroendocrine tumors: the rapid pace of no progress. Am J Gastroenterol 2007;102(7):1464-73.

[54] Lepage C, Bouvier AM, Manfredi S, Dancourt V, Faivre J. Incidence and management of primary malignant small bowel cancers: a well-defined French population study. Am J Gastroenterol 2006;101(12):2826-32.

[55] Gustafsson BI, Siddique L, Chan A, Dong M, Drozdov I, Kidd M, et al. Uncommon cancers of the small intestine, appendix and colon: an analysis of SEER 1973-2004, and current diagnosis and therapy. Int J Oncol 2008;33(6):1121-31.

[56] Haselkorn T, Whittemore AS, Lilienfeld DE. Incidence of small bowel cancer in the United States and worldwide: geographic, temporal, and racial differences. Cancer Causes Control 2005;16(7):781-7.

[57] Halfdanarson TR, Rubin J, Farnell MB, Grant CS, Petersen GM. Pancreatic endocrine neoplasms: epidemiology and prognosis of pancreatic endocrine tumors. Endocr Relat Cancer 2008;15(2):409-27.

[58] Kwekkeboom DJ, Krenning EP, Scheidhauer K, Lewington V, Lebtahi R, Grossman A, et al. ENETS Consensus Guidelines for the Standards of Care in Neuroendocrine Tumors: somatostatin receptor imaging with (111)In-pentetreotide. Neuroendocrinology 2009;90(2):184-9.

[59] Sundin A, Vullierme MP, Kaltsas G, Plöckinger U. ENETS Consensus Guidelines for the Standards of Care in Neuroendocrine Tumors: radiological examinations. Neuroendocrinology 2009;90(2):167-83.

[60] Yao JC, Eisner MP, Leary C, Dagohoy C, Phan A, Rashid A, et al. Population-based study of islet cell carcinoma. Ann Surg Oncol 2007;14(12):3492-500.

[61] Halfdanarson TR, Rabe KG, Rubin J, Petersen GM. Pancreatic neuroendocrine tumors (PNETs): incidence, prognosis and recent trend toward improved survival. Ann Oncol 2008;19(10):1727-33.

[62] Pieterman CR, Vriens MR, Dreijerink KM, van der Luijt RB, Valk GD. Care for patients with multiple endocrine neoplasia type 1: the current evidence base. Fam Cancer 2011;10(1):157-71.

[63] Maher ER, Neumann HP, Richard S. von Hippel-Lindau disease: a clinical and scientific review. Eur J Hum Genet 2011; 19(6):617-23.

[64] Thakker RV, Newey PJ, Walls GV, Bilezikian J, Dralle H, Ebeling PR, et al. Clinical practice guidelines for multiple endocrine neoplasia type 1 (MEN1). J Clin Endocrinol Metab 2012;97(9):2990-3011.

[65] Crocetti E, Paci E. Malignant carcinoids in the USA, SEER 1992-1999. An epidemiological study with 6830 cases. Eur J Cancer Prev 2003;12(3):191-4. 